\title{
Bandwidth Exchange for Enabling Forwarding in Wireless Access Networks
}

\author{
Dan Zhang*, Ryoichi Shinkuma*† and Narayan Mandayam* \\ ${ }^{*}$ WINLAB, Rutgers University \\ 671 Route 1 South, North Brunswick, NJ 08902 \\ Email: \{bacholic, shinkuma, narayan\}@winlab.rutgers.edu \\ ${ }^{\dagger}$ Graduate School of Informatics, Kyoto University \\ Yoshida-honmachi, Sakyo-ku, Kyoto, 606-8501, Japan \\ Email: shinkuma@i.kyoto-u.ac.jp
}

\begin{abstract}
Cooperative forwarding in wireless networks has shown to yield benefits of rate and diversity gains, but it needs to be incentivized due to the energy and delay costs incurred by individual nodes in such cooperation. In this paper we consider an incentive mechanism called Bandwidth Exchange $(B E)$ where the cooperating nodes flexibly exchange the transmission bandwidth (spectrum) as a means of providing incentive for forwarding data. The advent of cognitive radios with the ability to flexibly change their carrier frequency as well as their transmission bandwidth makes this form of incentive particularly attractive compared to other incentive mechanisms that are often based on abstract notions of credit and shared understanding of worth. Specifically, we consider a $N$-node wireless network and use a Nash Bargaining Solution $(N B S)$ mechanism to study the benefits of $B E$ in terms of rate and coverage gains.
\end{abstract}

\section{INTRODUCTION}

Cooperative forwarding is an essential technique to enhance connectivity and throughput for wireless networks. However, forwarding always incurs some sort of cost - either real costs like energy and power, or opportunistic cost like delay. To circumvent the above difficulties, we have recently proposed Bandwidth Exchange $(B E)$ as an incentive for forwarding. Specifically in [1], we have considered a two-node network where each node is endowed with orthogonal frequency resources and shown that a Nash Bargaining Solution (NBS) based mechanism can provide incentive for forwarding. In this paper we consider the extension of $B E$ to a $N$-node network, i.e., whenever a node asks another node for cooperation, it delegates a portion of its frequency resource to the forwarder as immediate compensation for the forwarder's cost. A similar $N B S$-based cooperation strategy for a two-node network was also discussed in [2]. Recent advances in cognitive radio have made this approach feasible. In particular, OFDMA (Mobile WiMAX) [3] and SC-FDMA (LTE) [4] technologies allow nodes to flexibly acquire and relinquish a number of the subcarriers/subchannels. Such spectrum agility achieved in radio technology has great promise to cope with the forwarding incentive problem that is studied in this paper.

\section{System Model And BAndwidth EXChange}

Consider $N$ nodes (labeled $1,2, \ldots, N$ ) communicating to an access point ( $A P$, labeled as node 0$)$ as shown in Fig.
1. Each node is assigned a nonoverlapping, hence orthogonal bandwidth $W_{i}$. The transmission power $P_{i}^{\mathrm{t}}$ for each node is fixed. The minimum required rate for each node is $R_{i}^{\min }$. We assume an ergodic fading model where the transmission is slotted and the channel gain $\rho_{i j}\left(=\rho_{j i}\right)$ in each slot is quasi-static and is an independent realization of a random variable (with marginal distribution $\left.p\left(\rho_{i j}\right)\right)$. To avoid confusion, subscript $i j$ always implies the direction from $i$ to $j$. If such a subscript is used in a transmission scheme, it is understood that $i$ is the source and $j$ is the forwarder (or the $A P$ if $j=0$ ).

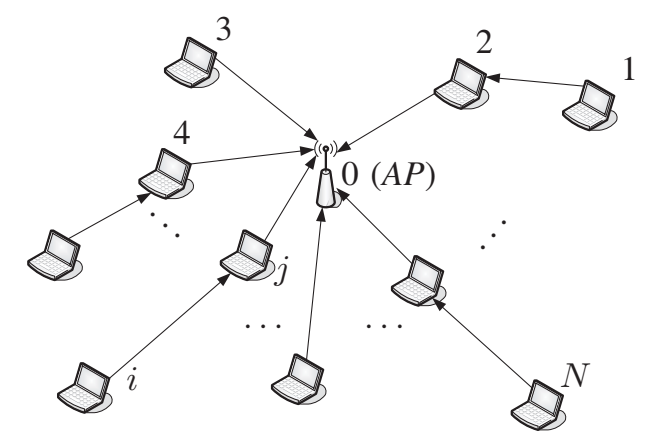

Fig. 1. Nodes connect to $A P$ through cooperative forwarding.

In every slot a node $i$ first attempts to transmit directly to the $A P$. If the direct link is under outage, i.e., the link capacity is smaller than the minimum required rate of node $i$, it tries to find a node $j$ which could help forward its data to $A P$, by means of $B E$. During transmission, node $i$ makes use of its available bandwidth up to $W_{i}$ as dictated by $B E$. The instantaneous direct link capacity $R_{i j}^{\text {ins }}$ from node $i$ to node $j$ in a slot is a function of node $i$ 's available bandwidth and $\rho_{i j}$ with transmission power $P_{i}^{\mathrm{t}}$ a fixed parameter. We assume there is no flow splitting and every forwarder serves at most one source.

The basic idea of cooperation through $B E$ is the source delegating a portion of the frequency band to the forwarder in exchange for cooperation that guarantees the minimum required rate of the source. When node $i$ transmits to the $A P$ directly, the resulting (noncooperation) rate is denoted as $R_{i}^{\mathrm{n}}=R_{i 0}^{\mathrm{ins}}\left(W_{i}, \rho_{i 0}\right)$. When node $j$ forwards data for node $i$ through $B E$, the resulting rate for $j$ is denoted as $R_{i j}^{\mathrm{r}}$ and $R_{i j}^{\mathrm{s}}$ 


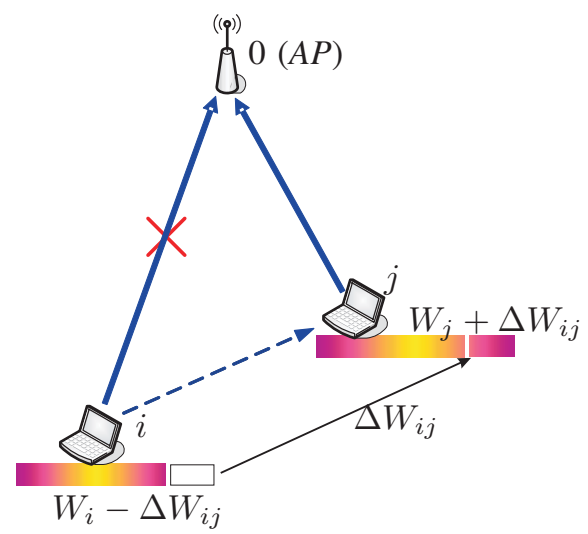

Fig. 2. When the direct link is under outage, node $i$ tries to solicit cooperation by delegating $\Delta W_{i j}$ to node $j$.

for the source node $i$. Note that $R_{i j}^{\mathrm{s}}=R_{i}^{\mathrm{min}}$ since a source only seeks to maintain the minimum required rate to connect to $A P$. Therefore, as shown in Fig. 2, the source node $i$ can withhold $W_{i}-\Delta W_{i j}$ and delegate $\Delta W_{i j}$ to the forwarder $j$ such that

$$
R_{i}^{\min }=R_{i j}^{\mathrm{ins}}\left(W_{i}-\Delta W_{i j}, \rho_{i j}\right) .
$$

Node $j$, in addition to guaranteeing $R_{i}^{\text {min }}$ for node $i$, uses the remaining capacity achieved with increased bandwidth $W_{j}+$ $\Delta W_{i j}$ for its own data,

$$
R_{i j}^{\mathrm{r}}=R_{j 0}^{\mathrm{ins}}\left(W_{j}+\Delta W_{i j}, \rho_{j 0}\right)-R_{i}^{\mathrm{min}} .
$$

Equations (1) and (2) define the basic mechanism of $B E$ as described in [1] - instead of raising transmission power, cooperation is achieved by autonomously reallocate bandwidth resources among the nodes. Note that they also describe the relationship of the rates $\left(R_{i j}\right)$ and bandwidth portion $\left(\Delta W_{i j}\right)$ to the link gain $\rho_{i j}$. For simplicity in notation, we suppress the explicit dependence. Link $i j$ is considered as under outage when it is too weak,

$$
\Delta W_{i j}<0
$$

or when it leads to outage for the forwarder,

$$
R_{i j}^{\mathrm{r}}<R_{j}^{\min }
$$

In either case, we also say the request from $i$ is not supportable at $j$.

\section{CoOperation Forwarding InCEnTED By $B E$}

In a fading environment, the role of a node as a forwarder or source can change from slot to slot. The decision made in a slot should take the consequences it entails in future slots into consideration. This situation is better modeled with an infinitely repeated game [1] [5], each slot corresponding to a stage game. A node $i$ under outage in a slot will request for cooperation through $B E$; a potential forwarder $j$ has to make a decision from a binary strategy space, i.e., to cooperate or not. Node $j$ will make a trivial decision to simply reject cooperation if the request is not supportable. Otherwise node $j$ will choose to cooperate with a nonzero probability to be discussed shortly.

The utility function $u_{i j}^{\text {ins }}$ of a stage game for an arbitrary node $j$, called instantaneous rate gain, is defined to be the rate increase achieved in that slot compared to noncooperation. Instantaneous rate gain is closely related to the strategy a node takes. For example, the instantaneous rate gain of node $j$ when it chooses to forward for node $i$ is

$$
u_{j}^{\mathrm{ins}}=u_{i j}^{\mathrm{ins}}=R_{i j}^{\mathrm{r}}-R_{j}^{\mathrm{n}},
$$

while

$$
u_{i}^{\text {ins }}=R_{i}^{\min } .
$$

The utility function of the repeated game for an arbitrary node $j$ is the average rate gain. A trivial stage game for node $j$ is one in which every decision involving $j$, no matter whether it is the source or forwarder, is trivial, then $u_{j}^{\text {ins }}=0$ for that stage. Therefore we focus only on nontrivial stage games and disregard, for each node $j$, those stage games that are trivial to $j$. In other words, in each nontrivial stage game of $j$, either $j$ is a source and sends a supportable request to some node $i$, or $j$ is a potential forwarder and receives a supportable request from some node $i$.

\section{A. Simplified N-Node Bargaining and Selection Policy}

In a two-node network, a nontrivial stage game consists of either node 1 sending a supportable request to node 2 , or node 2 sending a supportable request to node 1 . Suppose the two events happen with probability $P_{12}$ and $P_{21}$, respectively. A potential forwarder thus has two possible strategies: $C$ for cooperation and $N$ for noncooperation. The two-node $N B S$ (see [1]) tells us the probability $P_{12}^{\mathrm{c}}$ that node 2 chooses $C$ and the probability $P_{21}^{\mathrm{c}}$ that node 1 chooses $C$. Let $v_{1}, v_{2}$ denote the average rate gains of node 1 and 2 , respectively, in a nontrivial stage game. Further, let $\lambda_{1}, \lambda_{2}, \lambda_{3}, \lambda_{4}$ denote the mixing probabilities corresponding to joint strategies $\langle N, C\rangle,\langle C, C\rangle,\langle C, N\rangle,\langle N, N\rangle$. The $N B S$ is given as

$$
\begin{array}{ll}
\underset{\lambda_{1}, \lambda_{2}, \lambda_{3}, \lambda_{4}}{\operatorname{maximize}} & v_{1} v_{2}, \\
\text { subject to } & v_{1}=\lambda_{1} P_{21} R_{2}^{\mathrm{min}}+\lambda_{2}\left(P_{21} R_{2}^{\mathrm{min}}+P_{12} u_{12}\right) \\
& +\lambda_{3} P_{12} u_{12}+\lambda_{4} \cdot 0 \\
& v_{2}=\lambda_{1} P_{21} u_{21}+\lambda_{2}\left(P_{12} R_{1}^{\mathrm{min}}+P_{21} u_{21}\right) \\
& +\lambda_{3} P_{12} R_{1}^{\mathrm{min}}+\lambda_{4} \cdot 0 \\
& \lambda_{1}+\lambda_{2}+\lambda_{3}+\lambda_{4}=1 \\
& \lambda_{i} \geq 0, \quad i=1,2,3,4
\end{array}
$$

Then, the desired cooperation probabilities are

$$
P_{12}^{\mathrm{c}}=\lambda_{1}+\lambda_{2}, \quad P_{21}^{\mathrm{c}}=\lambda_{2}+\lambda_{3} .
$$

In the case of a $N$-node network, formulating the $N$-node $N B S$ is practically infeasible. One reason is that the strategy space for each node contains an exponentially increasing number of strategies relative to the number of nodes. This prompts us to look for approximate solutions with much lower complexity. 
One such solution is based on restricting cooperations to twohop forwarding. In other words, every node can reach the $A P$ via at most one other node. Since we have required one forwarder for one source and no flow splitting, eventually cooperation happens only between a distinct pair of nodes. It is then natural to approximate the $N$-node bargaining with a series of two-node bargainings, each completely disregarding the existence of other nodes and their influence. Consequently, the simplified $N$-node bargaining boils down to every node under outage in a slot carrying out independent two-node bargainings with every other node according to the two-node $N B S$ given in equation (7).

However, with this simplification, a potential forwarder may receive multiple cooperation requests while a source may receive multiple positive acknowledgements. A natural choice is to dictate that one of the source nodes is chosen by the forwarder with a probability proportional to the corresponding cooperation probabilities calculated from the twonode $N B S$ in equation (7). Specifically, suppose the requests from $i_{1}, i_{2}, \ldots, i_{k}$ are all supportable at forwarder $j$ and the corresponding cooperation probabilities calculated from the two-node $N B S$ are $P_{i_{1} j}^{\mathrm{c}}, P_{i_{2} j}^{\mathrm{c}}, \ldots, P_{i_{k} j}^{\mathrm{c}}$, then $j$ chooses to cooperate with $i_{\ell}(1 \leq \ell \leq k)$ with probability

$$
P_{i_{\ell} j}^{\prime \mathrm{c}}=\frac{P_{i_{\ell} j}^{\mathrm{c}}}{P_{i_{1} j}^{\mathrm{c}}+P_{i_{2} j}^{\mathrm{c}}+\cdots P_{i_{k} j}^{\mathrm{c}}} .
$$

On the other hand, a source may also receive positive responses from multiple forwarders. The source then randomly chooses one to follow as every forwarder equally guarantees the minimum required rate of the source. It is possible for the forwarders whose offers are turned down to pick up requests from other sources that they can help but we do not pursue this situation and instead simply assume this concludes the bargaining phase of the slot. Nodes then use the remaining time of the slot to either cooperate, or to transmit on their own, or to stay off connection.

\section{NuMERICAL RESUlTS}

\section{A. Simulation model}

Our mechanism is applicable to any multihop network, infrastructured or ad hoc, in a licensed or unlicensed band. For the purpose of illustration, we consider an OFDMA [3] transmission scheme much like the one used in mobile WiMAX [6]. The presence of orthogonal subcarriers in an OFDMA system provides a natural platform for implementing $B E$ by exchanging orthogonal frequency bands.

We simulate a slotted system using parameters that are typical to mobile WiMAX. Each node is pre-assigned $20 \mathrm{dBm}$ fixed transmit power [7] [8] and $500 \mathrm{kHz}$ transmission bandwidth corresponding to 50 subcarriers at $10 \mathrm{kHz}$ spacing. When a node delegates bandwidth, it transfers a number of the subcarriers to a forwarder. Since nodes in our network use mutually orthogonal portions of frequency, we model the instantaneous capacity of link $i j$ using its information- theoretic rate

$$
R_{i j}^{\mathrm{ins}}\left(W, \rho_{i j}\right)=W \log _{2}\left(1+\frac{\rho_{i j} P_{i}^{\mathrm{t}}}{W}\right), \quad i, j=0,1, \ldots, N .
$$

Links are under independent Rayleigh fading and the link gain in each slot is an independent realization of a Rayleigh random variable. Equivalently, this implies that $\rho_{i j}$ is exponentially distributed

$$
p\left(\rho_{i j}\right)=\frac{1}{\bar{\rho}_{i j}} \exp \left(-\frac{\rho_{i j}}{\bar{\rho}_{i j}}\right)
$$

where the statistical mean $\bar{\rho}_{i j}$ is given by the path loss model

$$
\bar{\rho}_{i j}=\kappa d^{-3}, \quad\left(\kappa=6 \times 10^{6} \mathrm{MHz} \cdot \mathrm{m}^{3} / \mathrm{mW}\right) .
$$

The above simulation model implicitly assumes that the average rate of a transmission is one that is obtained when all the subcarriers used undergo identical fading. This is done for the simplicity of illustration but the idea of $B E$ and its applicability to frequency selective OFDMA systems is still valid. The pairwise $N B S$ with $B E$ in equation (7) is implemented for the above channel model. We simulate for sufficiently many slots to assess the average performance.

\section{B. A Three-Node Example}

We first present a three-node example to show the power of $B E$ with $N B S$ in improving coverage and rate. Suppose node 1 is fixed at $(-450 \mathrm{~m}, 0)$ and node 2 at $(450 \mathrm{~m}, 0)$. Node 3 is allowed to vary its location in a $2000 \times 2000 \mathrm{~m}^{2}$ region as shown in Fig. 3.

If we set the minimum required rate for each node to $700 \mathrm{kbps}$ and the tolerable outage probability to $10 \%$, Fig. 3(a) shows coverage area for node 3 without cooperation. Fig. 3(b) shows the improvement in coverage area achieved when using $B E$ with $N B S$ for the same level of outage. Fig. 3(c) shows a comparable coverage area for node 3 in the absence of cooperation. However, the minimum required rate now has to be lowered to $300 \mathrm{kbps}$ to generate an identical level of outage. This simple illustration indicates that $B E$ can be used to either increase coverage, or increase the supported rate.

\section{Performance Evaluation in a N-Node Network}

In this section, we present a comparative evaluation of $B E$ with $N B S$ with noncooperation, as well as with a simple heuristic cooperative forwarding scheme. Specifically, the simple heuristic requires that in each slot, every potential forwarder tries to cooperate with a supportable source (see equations (3) and (4)) randomly and the source also randomly follows a cooperating forwarder. This is equivalent to setting $P_{i j}^{\mathrm{c}}=P_{j i}^{\mathrm{c}}=1$ in the $N B S$. Recall that every node has its own bandwidth. When a node under outage cannot get any cooperation its bandwidth will go unused in that slot. The simple heuristic is a straightforward measure to reduce, if not minimize, such waste. Therefore it serves as a good benchmark for the average rate gain and spectral efficiency to be addressed. 


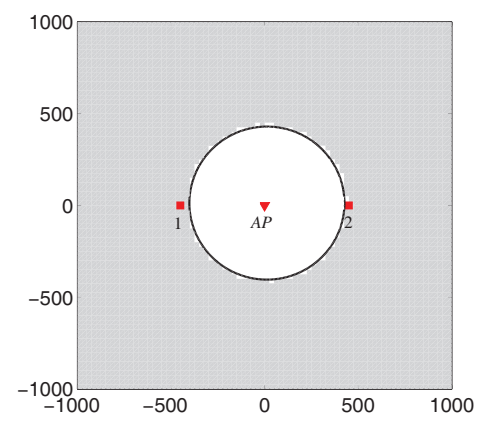

(a)

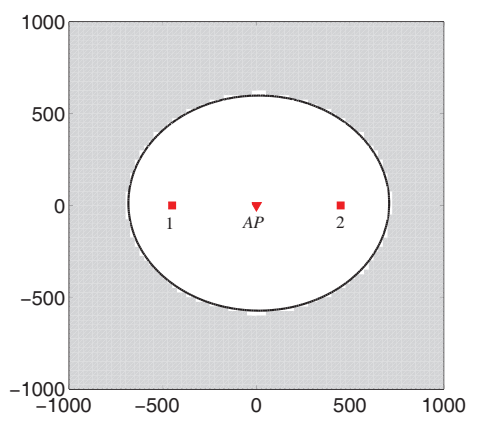

(b)

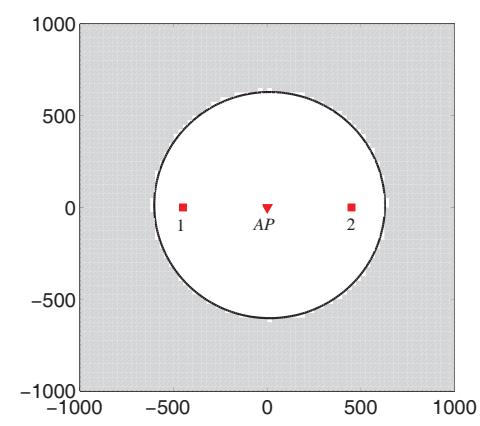

(c)

Fig. 3. Improvement in coverage and rate $-P_{3}^{\text {out }}<0.1$ in the white area. (a):noncooperation, $R_{i}^{\mathrm{min}}=700 \mathrm{kbps}$; (b): $B E$ with $N B S, R_{i}^{\mathrm{min}}=700 \mathrm{kbps}$; (c):noncooperation, $R_{i}^{\min }=300 \mathrm{kbps} ; i=1,2,3$.

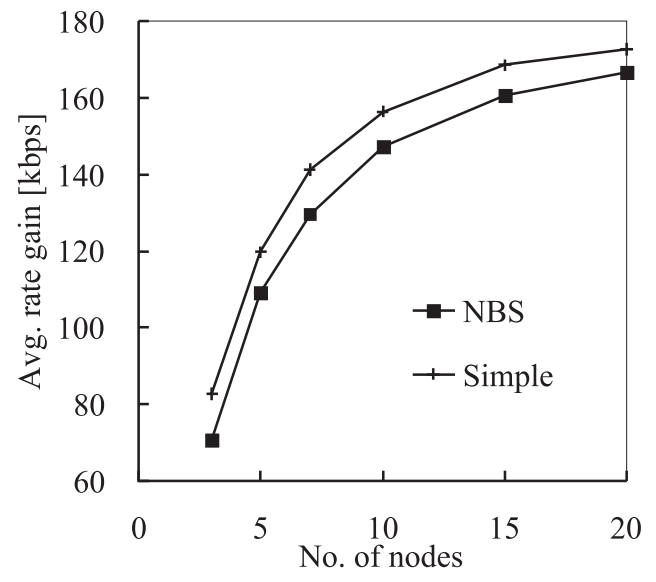

Fig. 4. Average rate gain in a cell consisting of varied number of nodes.

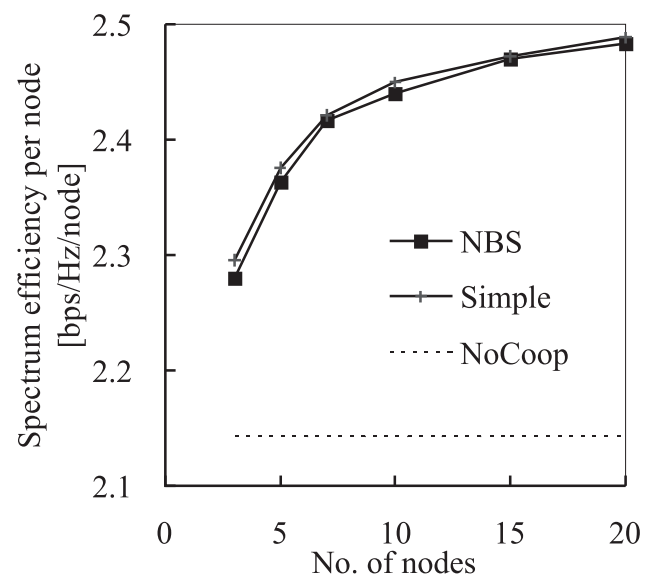

Fig. 5. Spectrum efficiency per node.
As mentioned earlier, we simulate a slotted system that uses parameters typical to mobile WiMAX. We consider up to 20 nodes randomly placed in a cell with a radius of $1000 \mathrm{~m}$. Our results are obtained by averaging over multiple time slots and location instantiations of mobiles. Specifically, we look at the metrics of average rate gain, spectrum efficiency and fairness as a function of the number of nodes in the system.

1) Average Rate Gain: Fig. 4 shows the average rate gain available to any node in a cell when the minimum required rate is set to $700 \mathrm{kbps}$. No matter which algorithm is used, the average rate gain is an increasing function of the number nodes in the system, illustrating the benefits of user cooperation diversity. The simple heuristic exhibits the best performance thanks to its generous nature, though only the nodes far away from the $A P$ are the real beneficiaries while nodes close to the $A P$ usually suffer substantial loss while forwarding for others.

2) Spectrum Efficiency: Fig. 5 shows the spectrum efficiency averaged over the number of nodes to illustrate the effect of user cooperation diversity when the minimum required rate is set to $700 \mathrm{kbps}$. Note that in our model, nodes are employing orthogonal subcarriers and hence do not interfere with each other. However, the spectrum efficiency per node increases with the number of nodes. Noncooperation performs well below the two cooperative strategies. It is noteworthy that $B E$ with $N B S$ has almost the same spectrum efficiency with the very generous simple heuristic algorithm.

3) Geometric Mean of Rate Gains: The NBS does not take average rate gain or spectrum efficiency as an explicit optimization objective. Rather, it provides a proportionally fair rate allocation, i.e., it tries to maximize the product of rate gains, or equivalently, the geometric mean of rate gains. In this sense, the geometric mean of rate gains can be regarded a measure of the average amount of individual incentive that a node has for cooperation when $B E$ is used with $N B S$. Let $\mathcal{I}$ denote the fairness metric defined as

$$
\mathcal{I}=\left(\prod_{i=1}^{N} \max \left(u_{i}, 0\right)\right)^{1 / N}
$$

The implication is that for fair cooperation schemes, the average rate gains are always positive and equation (13) reduces to the canonical geometric mean as an average amount of individual incentive. When the average rate gains for some nodes are negative, then equation (13) indicates $\mathcal{I}=0$, i.e., the 


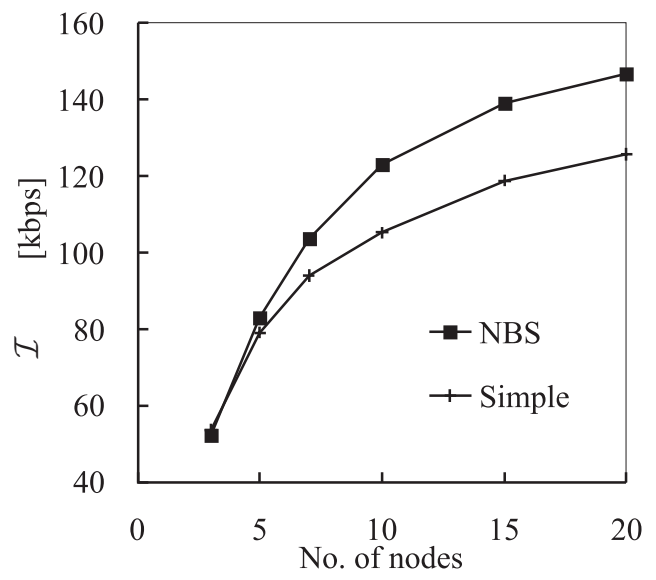

Fig. 6. Geometric mean of rate gains as a measure of fairness.

scheme is unfair. Fig. 6 shows $\mathcal{I}$ as a function of the number of nodes when the minimum required rate is set to $700 \mathrm{kbps}$. We observe that, as a proportionally fair cooperating scheme, $B E$ with $N B S$ performs desirably and the average amount of individual incentive increases with the number of nodes. This observation is consistent with user cooperation diversity increasing with the number of nodes. On the contrary, the noncooperation scheme has $\mathcal{I}=0$. The simple heuristic can be unfair as well. In fact, our experiments reveal in roughly $20 \%$ of simulation trials, one or more nodes experience negative rate gains.

\section{CONCLUSION AND Discussions}

In this paper we discussed a cooperative forwarding incentive mechanism called Bandwidth Exchange where relay nodes forward data in exchange for bandwidth that is delegated by source nodes. The advent of cognitive radios with the ability to flexibly change their carrier frequency as well as their transmission bandwidth makes this form of incentive particularly attractive. Further, the use of OFDMA or SC-FDMA based access allows for the flexible exchange of frequency bands among the nodes. Compared to other incentive mechanisms such simple bandwidth delegation provides more tangible and immediate incentive. Specifically, we considered a $N$-node wireless network and used a Nash Bargaining Solution to study the benefits of $B E$ in terms of rate and coverage gains. Further, the $N B S$ also assured that the rate allocations were proportionally fair.

While the results presented here showed the benefits of $B E$ as an incentive mechanism, an interesting and important aspect of $B E$ is the need for a distributed protocol for implementing the $N B S$ strategy in a $N$-node network. The choices of selection policies as well as other heuristic $B E$ based incentive mechanisms also need to be considered. These will be discussed in an upcoming paper [9].

\section{ACKNOWLEDGEMENT}

This work is supported in part by the NSF under grant number CCF-0634973 and CNS-0721826. The work is also supported in part by Japan Society for the Promotion of Science (JSPS) under Grant-in-Aid for Encouragement of Young Scientists (B) (no. 21760288).

\section{REFERENCES}

[1] D. Zhang, O. Ileri, and N. Mandayam, "Bandwidth exchange as an incentive for relaying," in 42nd Annual Conference on Information Sciences and Systems (CISS), Princeton, NJ, March 2008, pp. 749-754.

[2] Z. Zhang, J. Shi, H.-H. Chen, M. Guizani, and P. Qiu, "A cooperation strategy based on Nash Bargaining Solution in cooperative relay networks," IEEE Trans. Vehicular Technology, vol. 57, no. 4, pp. 2570-2577, July 2008.

[3] H. Yin and S. Alamouti, "OFDMA: A broadband wireless access technology," in IEEE Sarnoff Symposium, 2006, pp. 1-4.

[4] F. D. Calabrese, M. Anas, C. Rosa, P. E. Mogensen, and K. I. Pedersen, "Performance of a radio resource allocation algorithm for utran lte uplink," in IEEE VTC, April 2007, pp. 2895-2899.

[5] D. Fudenberg and J. Tirole, Game Theory. MIT Press, 1991.

[6] "IEEE standard for local and metropolitan area networks part 16: Air interface for fixed and mobile broadband wireless access systems amendment 2: Physical and medium access control layers for combined fixed and mobile operation in licensed bands and corrigendum 1," IEEE Std 802.16e-2005 and IEEE Std 802.16-2004/Cor 1-2005 (Amendment and Corrigendum to IEEE Std 802.16-2004), pp. 0_1-822, 2006.

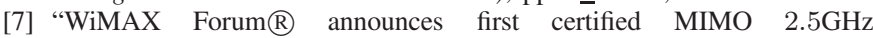
mobile WiMAX products," June 2008, [Online]. Available: http://www.wimaxforum.org/news/pr/view?item_key=cffca4e77e1900b8 fa727fe754a60be0db849e6.

[8] “XOHM ExpressCard," [Online]. Available: http://www.xohm.com /en_US/shop/devices/pccard-samsung-swce100.html.

[9] D. Zhang, R. Shinkuma, and N. Mandayam, "Bandwidth exchange: An energy conserving forwarding mechanism for FDMA networks," (in preparation). 